\title{
PACIFIC: Time for a surgical IIIA uprising
}

\author{
Harvey I. Pass, MD
}

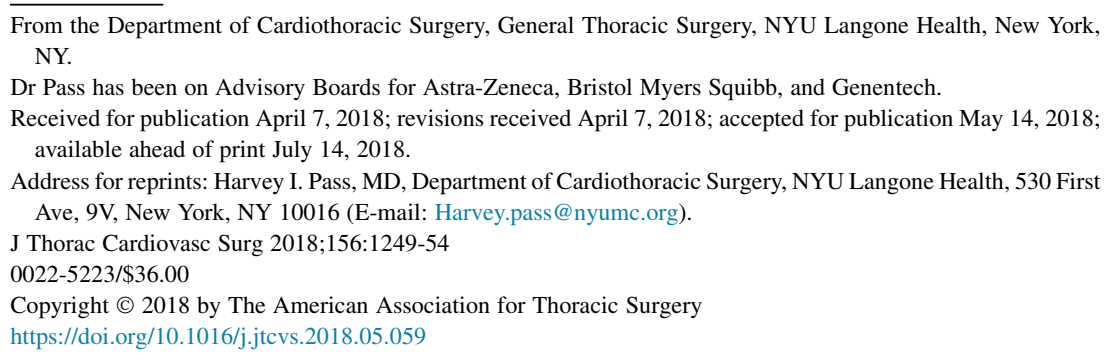

From the Department of Cardiothoracic Surgery, General Thoracic Surgery, NYU Langone Health, New York, NY.

Dr Pass has been on Advisory Boards for Astra-Zeneca, Bristol Myers Squibb, and Genentech.

Received for publication April 7, 2018; revisions received April 7, 2018; accepted for publication May 14, 2018; available ahead of print July 14, 2018.

Address for reprints: Harvey I. Pass, MD, Department of Cardiothoracic Surgery, NYU Langone Health, 530 First Ave, 9V, New York, NY 10016 (E-mail: Harvey.pass@nyumc.org).

J Thorac Cardiovasc Surg 2018;156:1249-54

$0022-5223 / \$ 36.00$

Copyright (C) 2018 by The American Association for Thoracic Surgery

https://doi.org/10.1016/j.jtcvs.2018.05.059

Feature Editor's Note-If you are not already committed based on the title alone, this is an article that should be read by all thoracic surgical oncologists. The PACIFIC trial is randomized trial recently published in the New England Journal of Medicine that showed improvement in progression-free survival in patients with unresectable stage IIIA-B non-small cell lung cancer treated with PD-L1 blockade (compared with placebo) after chemoradiotherapy. Within the past year, it has become clear that immune checkpoint inhibitors will have important roles in the treatment of all stages of lung cancer, not just metastatic disease. The PACIFIC trial highlights the significant impact that checkpoint blockade has on locally advanced non-small cell lung cancer, including stage IIIA disease. Subsequent published editorials by thought leaders in our field have extended the reach of these findings by suggesting that all stage IIIA non-small cell lung cancer is best treated nonsurgically with chemoradiotherapy followed by checkpoint blockade. It is important that thoracic surgeons remain conversant in this rapidly evolving field and should understand the nuances and surgical considerations of the PACIFIC trial so that they may be insightfully discussed with our colleagues in medical and radiation oncology. These nuances and considerations are thoughtfully and logically crystallized in this Feature Expert Opinion article, and presented in the context of the most clinically relevant elements of our current understanding of surgery for N2 disease. The reader will be empowered with data, inspired by wisdom, and energized to rise.

\section{Bryan M. Burt, MD}

Heterogeneous is the word used most to describe stage IIIA non-small cell lung cancer (NSCLC); the term could also describe the opinions regarding its management. In this era of personalized medicine, homogeneous therapy would certainly be a luxury for patients with locally advanced lung III NSCLC. ${ }^{2}$

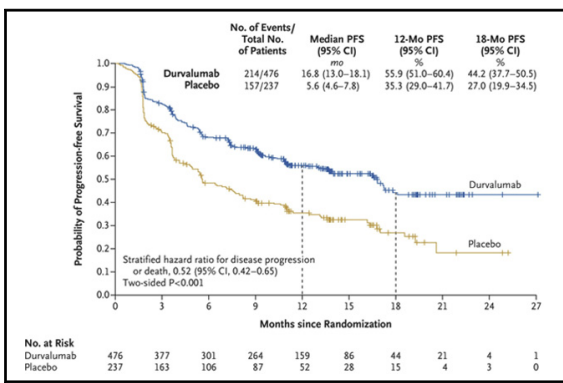

PACIFIC median PFS was increased by 11 months with adjuvant immunotherapy. Reprinted with permission

Central Message

The PFS results of PACIFIC in unresectable stage III NSCLC calls for novel, standalone trials exploring induction/adjuvant chemo/chemoRT with immunotherapy in surgically resectable IIIA patients.

cancer. In fact, if the thoracic surgical community carefully reads editorials in journals with a high impact factor, such as the New England Journal of Medicine, it would see that nonsurgical thought leaders are already hypothesizing that the future therapy of stage IIIA NSCLC may have been answered with the PACIFIC trial. ${ }^{1}$

The data from the PACIFIC study may end the debate about the role of surgery in patients with Stage III NSCLC, with durvalumab after chemoradiotherapy redefining best practice for all patients with Stage

The data from this trial could potentially define IIIA as a homogenenous entity with regard to therapy. It is not like thoracic surgeons have not already heard about the results of the PACIFIC trial, which has been summarized in the JTCVS in 2 previous expert opinions. ${ }^{3,4}$ Moreover, surgeons have embraced the role of immunotherapy for thoracic malignancies by their participation national adjuvant immunotherapy trials (Alchemist; https://clinicaltrials.gov/ ct2/show/NCT02595944?term=ALCHEMIST\&rank=3) and consortium-based window-of-opportunity trials, which include stages IB to IIIA (ML39236, Lung Cancer Mutation Consortium, Genentech, https://clinicaltrials.gov/ct2/results? cond $=\&$ term $=\mathrm{ml39236} \& \mathrm{cntry}=\&$ state $=\& \operatorname{city}=\&$ dist $=$ $\&$ Search=Search; Checkmate 816, Bristol-Myers Squibb, 
https://clinicaltrials.gov/ct2/results? cond $=\&$ term $=$ check mate $+816 \&$ cntry $=\&$ state $=\&$ city $=\&$ dist $=\&$ Search $=$ Search $)$. These trials, however, do not really address the issue that is raised in the editorial which implies that the surgeon's role in stage IIIA in the future may be limited to pretreatment staging and posttreatment salvage surgeries. My worry (full disclosure: I am a thoracic surgeon who evaluates and resects patients with stage IIIA disease) is that if consensus leaders and internationally renowned medical oncologists, immunotherapy leaders, and radiation oncologists say or write their opinion, such hypotheses could be taken as gospel by the nonsurgical congregation. The majority of thoracic surgeons attend multidisciplinary conferences where situations regarding IIIA disease are debated, and thoracic surgeons must be able to discuss the PACIFIC trial insightfully in the context of surgery for $\mathrm{N} 2$ or $\mathrm{T} 4$ disease, and to enlighten our colleagues about the newest data regarding (1) accumulating data that surgery for lung cancer before or after immunotherapy is safe and possibly efficacious, (2) exactly what are the present guidelines to consider surgery for $\mathrm{N} 2$ disease, (3) when to decide whether a stage IIIA patient is resectable or not, (4) the issue of restaging the patient, (5) the safety of induction therapy for stage IIIA disease as it now stands, and (6) matters of local and systemic control.

\section{THE PACIFIC TRIAL: INQUIRIES OF A CURIOUS SURGEON}

It goes without saying that PACIFIC is a landmark trial whose impact in NSCLC cannot be underestimated. The bottom line is that a 2:1 randomization of durvalumab after chemoradiation therapy was associated with an 11-month increase in progression-free survival (PFS) compared with chemoradiation and placebo in unresectable patients (Figure 1). What was defined as an unresectable patient?

"Eligible patients had histologically or cytologically documented stage III, locally advanced, unresectable NSCLC according to the Staging Manual in Thoracic Oncology, version 7, of the International Association for the Study of Lung Cancer." "The only problem is that in consultation with Dr Peter Goldstraw who was the editor of the staging manual, "nowhere in the 7th edition published by the IASLC [International Association for the Study of Lung Cancer] did we specify that some IIIA cases were defined as 'resectable' or 'unresectable"' (personal communication, March 28, 2018). However, all 713 patients were deemed unresectable by a thoracic surgeon before randomization. The New England Journal of Medicine provides a copy of the protocol, and a quick search for "surgeon" revealed no hits, and "surgery" related only to "isolated lesions," excluding "target lesions." Therefore, we must assume that this stage III trial used other criteria for unresectability than those specified in the National Comprehensive Cancer Network (NCCN) guidelines version (board-certified thoracic surgeon should determine the role of surgery before initiating therapy) or the European Society for Medical Oncology guidelines (" [u]nresectable ... refers to the situation that—even after induction therapy-a complete resection [R0] would not be possible, based on evaluation within a multidisciplinary team, including an experienced thoracic surgeon"). ${ }^{5}$ Fortunately, however, there is a subdivision of patient staging in the PACIFIC manuscript into IIIA and IIIB patients, but unfortunately one cannot tell whether there was an imbalance of patients with $\mathrm{N} 2$ or $\mathrm{T} 4$ disease within that subset because there is no mention of T-stage status within the manuscript. Nevertheless, it seems that the addition of adjuvant immunotherapy was equally beneficial in stage IIIA disease according to the subgroup analysis with a 0.53 hazard ratio of progression compared with placebo.

\section{SURGICAL RELEVANCE IN STAGE IIIA: AN EVOLUTION BUT FAR FROM PERFECT}

If thoracic surgeons want to be able to justify their role in the management of stage IIIA disease in the post-PACIFIC era, they need to not only defend this position with peer reviewed or consensus data, but also recognize that future stage IIIA neoadjuvant protocols must describe (and study) more precisely preoperative eligibility and intraoperative and postoperative results to eliminate the ambiguity described in PACIFIC. Thoracic surgeons themselves are aware that the surgical management of stage IIIA disease in 2018 is not the same as it was in 1994, when the oftenquoted as religion and frequently disparaged "lobectomy" subset analysis INT0139 trial began. ${ }^{6}$ With all of its faults, it is important to remember that in INT0139, the PFS was longer in the chemoradiotherapy and surgery group (median 12.8 months [interquartile range, 5.3-42.2] vs 10.5 months [interquartile range, 4.8-20.6]; hazard ratio, 0.77 [95\% confidence interval, 0.62-0.96]; $P=.017$ ), and PFS was $22 \%$ versus $11 \%$ for chemoradiotherapy and surgery versus chemoradiotherapy at 5 years. Nevertheless, since 2009 when INT0139 was published, issues regarding stratification of the stage IIIA surgical candidate, the need and dose for radiation therapy, the value of postinduction staging, and the safety of resection have been debated in the literature, leading to some consensus recommendations.

\section{Who Are the Best Surgical Stage IIIA Candidates?}

NCCN recommendations for stage IIIA surgical management with induction have attempted to quantify the degree of $\mathrm{N} 2$ involvement (including the " $3 \mathrm{~cm}$ single node" guideline). On closer inspection, this recommendation was adapted from the original report by Andre et al. ${ }^{7}$ regarding the influence of single and multistation N2 disease. Although more than $90 \%$ of queried NCCN surgeons agree with that NCCN recommendation, $47.6 \%$ of them would also consider surgery after induction in patients with multi-station N2 disease. Thoracic surgeons decide up front whether a stage 
IIIA patient will be a candidate for resection after induction ${ }^{8,9}$; however, posttherapy response assessment is a major consideration for final surgical selection (discussed later), especially since complete mediastinal adenectomy is mandated, if possible, at the time of resection. A more precise quantitative algorithm for choosing patients in the immunotherapy era will be essential before any consideration of abandoning a surgical approach should be considered, hopefully by some imaging or blood-based biomarker modality before therapy is initiated.

Perhaps improvements in imaging techniques will help thoracic surgeons to be more precise in their assessments of stage IIIA response in the immunotherapy era. This scenario of imaging before resection of stage IIIA disease is not new to thoracic surgeons, and it could become more complicated. Certainly, the role of preinduction and postinduction positron emission tomography (PET)-computed tomography (CT) standardized uptake value (SUV) has been controversial with recent reports stating that the presence of residual nodal disease is not predicted by PET-CT, ${ }^{8}$ whereas other studies report that less than $60 \%$ reduction in $\mathrm{N} 2$ SUVmax is more likely to reflect persistent N2 disease. ${ }^{10}$ The International Association for the Study of Lung Cancer has recently emphasized capturing prospective data not only on the site and number of N2 stations involved, but also the influence of concomitant $\mathrm{N} 1$ and $\mathrm{N} 2$ disease. ${ }^{11}$ Other novel approaches for quantification of $\mathrm{N} 2$ disease and prognosis include measurement of CT lymph node volume but might not predict posttreatment response. ${ }^{12}$ With all the refinement in candidate selection described here, an individual's candidacy as a surgical candidate may come down to whether, in the surgeon's opinion, the patient could have an $\mathrm{R} 0$ resection with complete mediastinal lymphadenectomy before treatment.

Thoracic surgeons in the immunotherapy era will also need to know that immune-related response criteria that existed in 2009 according to the World Health Organization ${ }^{13}$ have been updated as the immune Response Evaluation Criteria in Solid Tumors (iRECIST). ${ }^{14}$ iRECIST proposes unidimensional measurement of lesions ${ }^{15}$ and emphasis on consecutive evaluations to report unconfirmed progressive disease. Pseudoprogression, an initially detectable increase in tumor or nodal bearing sites, can be diagnosed only if tumor shrinkage is detected at subsequent assessments and is in fact a good prognostic sign. The occurrence of true pseudoprogression is a rare event in NSCLC. Even the platform that will be used in the future to evaluate immunotherapy responses may change, because not only the size of the lesions but the composition of targets and contrast enhancement by CT should be evaluated with novel techniques, including dual-energy contrast CT. PET-CT with new enhancers that can track T cell infiltration into tumors, including ${ }^{18} \mathrm{~F}-\mathrm{FAC},{ }^{18} \mathrm{~F}$-FAraG, and labeled monoclonal antibodies specific for $\mathrm{T}$ cell antigens including PD-L1, are now being investigated in humans. ${ }^{16}$
Although accurate noninvasive determination of immunotherapeutic responses is exciting, let us remember that the best way to quantitate whether those novel imaging techniques occur is by pathologic evaluation of the response in the tissue harvested by surgeons. In fact, immunotherapy responses have already been reported in patients having lung cancer resection after immunotherapy. Chaft et al. ${ }^{17}$ reported a $41 \%$ major pathologic response $(\leq 10 \%$ viable tumor cells) in 21 patients (7/21 stage IIIA) having surgery after induction nivolumab without operative mortality. ${ }^{17}$ The need for better imaging predictors of response is emphasized by these investigators in that these pathologic responses were independent of radiologic findings or PDL1 status at pretreatment.

\section{CAVEAT 1}

Thoracic surgeons, independent of PFS and overall survival in studies of immunotherapy and stage IIIA lung cancer, provide the best way to analyze treatment response to immunotherapy at this time through an R0 resection, which in the "targeted therapy" era was known as "tissue is the issue."

\section{The Issue of Surgical Restaging: Does It Matter When?}

There is no doubt that residual N2 disease after definitive therapy influences survival, and whether restaging should influence subsequent surgical management is not at all standardized, even by leaders in the field. ${ }^{8,9,18}$ Some will only restage after therapy "if there is some concern that the patient may not be a good operative candidate or we think there is persistent multistation, bulky disease that may not be resectable," ${ }^{, 8}$ while others who associated improved survival with restaging "suggest that pathologic restaging allowed for more appropriate selection of patients for surgery." ${ }^{19}$ Alternatively, why do mediastinal staging at all? A recent editorial suggests that if PET-CT reveals only ipsilateral $\mathrm{N} 2$ disease, and the tumor and lymph nodes are resectable as defined by "non-fixed and non-bulky N2 disease," then invasive staging is "irrelevant." 20

While thoracic surgeons argue about the appropriateness of restaging before a potential $\mathrm{R} 0$ resection, medical and radiation oncologists image and reimage until it is time to document the recurrence by endobronchial ultrasound or surgery. Bluntly stated, who knows how many patients have residual disease in lymph nodes after chemoradiation until they recur locally? According to PACIFIC, the incidence of progression in the lung or lymph nodes was decreased by the immunotherapy from $17 \%$ to $12 \%$ and $11 \%$ to $6 \%$, respectively. ${ }^{1}$ It is of interest that in the original INT0913 trial, primary tumor site and lymph node recurrence rates were $2 \%$ and $7 \%$, respectively. ${ }^{6}$ Similar reductions in locoregional recurrence rates when surgery is part of the induction package have been seen in large, 


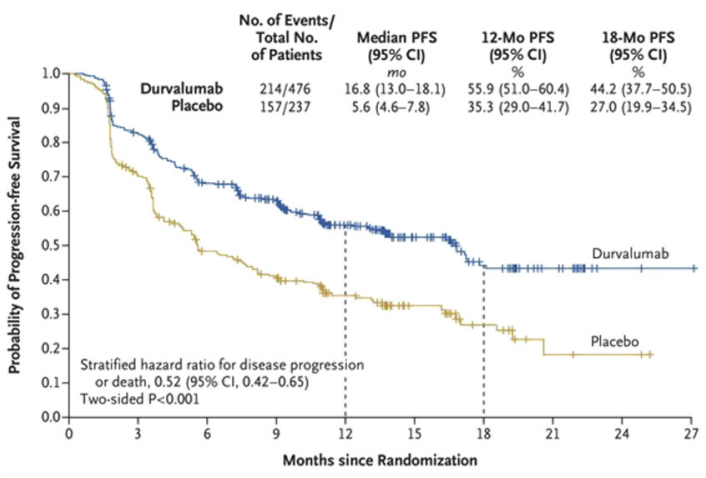

$\begin{array}{lrrrrrrrrrr}\text { No. at Risk } & & & & & & & & & \\ \text { Durvalumab } & 476 & 377 & 301 & 264 & 159 & 86 & 44 & 21 & 4 & 1 \\ \text { Placebo } & 237 & 163 & 106 & 87 & 52 & 28 & 15 & 4 & 3 & 0\end{array}$

FIGURE 1. Progression-free survival in the intention-to-treat population. Shown are Kaplan-Meier curves for progression-free survival (PFS), defined according to the Response Evaluation Criteria in Solid Tumors, version 1.1, and assessed by means of a blinded independent central review. Tick marks indicate censored observations, and vertical lines indicate the times of landmark PFS analyses. The intention-to-treat population included all patients who underwent randomization. $C I$, Confidence intervals. From Antonia SJ, Villegas A, Daniel D, Vicente D, Murakami S, Hui R, et al. Durvalumab after chemoradiotherapy in stage III non-small cell lung cancer. N Engl J Med. 2017;377:1919-29. Copyright (c) 2017 Massachusetts Medical Society. Reprinted with permission from Massachusetts Medical Society.

single-institution studies, ${ }^{21,22}$ In addition, in the recent Memorial Sloan Kettering Cancer Center series of 100 stage IIIA patients having surgery after induction chemotherapy, there was a $7 \%$ rate of isolated locoregional recurrence. ${ }^{8}$ Obviously, locoregional recurrence rates will depend on the residual cancer in the mediastinum after induction, and the decrease in PACIFIC of locoregional recurrence using postchemoradiotherapy immunotherapy mirrors the decrease in the recurrence dynamics seen when surgery is part of a trimodality or bimodality package. Is it not reasonable (or at least "hypothesis testing") to think that the addition of immunotherapy after induction chemo/chemoradiotherapy would only add to the length of PFS in patients selected for surgery? Moreover, caveat 1 comes into play again, because an induction regimen of chemotherapy and immunotherapy followed by surgery could influence whether to use postoperative radiotherapy (PORT) if the mediastinum has greater than $10 \%$ residual disease. ${ }^{23,24}$ Certainly, such a hypothesis should be tested in a clinical trial in which patients with residual disease after induction and surgical resection of stage IIIA disease are randomized to receive either PORT or PORT and maintenance immunotherapy.

\section{CAVEAT 2}

Surgery does well in regard to local recurrence rates compared with radiation. Induction therapy alone with immunotherapy may be a logical induction strategy in the future, reserving radiation for postoperative residual disease or close margins.

\section{Safety of Stage IIIA Surgery}

In the immunotherapy era, the role of pretreatment tissue biomarkers such as PDL1 or surrogate blood-based biomarkers may be just as important in the prediction of residual disease if induction therapy is to be given as part of the regimen. A handful of trials already address this issue and comment on the safety of thoracic surgery in patients treated with checkpoint inhibitors, which is an important issue because safety of surgical resection in stage IIIA disease has always been an argument for chemoradiation

TABLE 1. International trials of immunotherapy specifically in resectable or unresectable stage III non-small cell lung cancer

\begin{tabular}{|c|c|c|c|c|}
\hline Trial/sponsor & Phase & NCT & Stage & Protocol \\
\hline $\begin{array}{l}\text { Swiss Group for Clinical } \\
\text { Cancer Research }\end{array}$ & II & NCT02572843 & IIIA resectable & $\begin{array}{l}\text { Durvalumab neoadjuvant with cisplatinum docetaxel and } \\
\text { adjuvant }\end{array}$ \\
\hline Spanish Lung Cancer Group & II & NCT03081689 & IIIA resectable & Nivolumab neoadjuvant with carbo-taxol then adjuvant \\
\hline $\begin{array}{l}\text { European Thoracic Oncology } \\
\text { Platform and BMS }\end{array}$ & II & NCT02434081 & IIIA & $\begin{array}{l}\text { Nivolumab combination with standard first-line } \\
\text { chemotherapy and radiotherapy in locally advanced stage } \\
\text { IIIA/B non-small cell lung carcinoma (NICOLAS) }\end{array}$ \\
\hline $\begin{array}{l}\text { Centre hospitalier de l'Université } \\
\text { de Montréal }\end{array}$ & $\mathrm{I} / \mathrm{II}$ & NCT02733250 & IIIA & $\begin{array}{l}\text { A phase I/II study of pembrolizumab in combination with } \\
\text { nab-paclitaxel in patients with unresectable stage III or } \\
\text { stage IV non-small cell lung carcinoma }\end{array}$ \\
\hline Case Western & I & NCT02987998 & IIIA resectable & $\begin{array}{l}\text { A phase I safety and feasibility study of neoadjuvant } \\
\text { chemoradiation plus pembrolizumab followed by } \\
\text { consolidation pembrolizumab in resectable stage } 3 \text { A non- } \\
\text { small cell lung cancer }\end{array}$ \\
\hline University of Turin, Italy & II & NCT03379441 & IIIA & $\begin{array}{l}\text { Pembrolizumab (MK-3475) as maintenance in treated } \\
\text { patients with unresectable stage III non-small cell lung } \\
\text { cancer (MP-LALC) }\end{array}$ \\
\hline
\end{tabular}


therapy by a cadre of nonsurgical care providers. The original postoperative mortality of chemoradiotherapy and surgery published in INT0139 of $8 \%$ is no longer applicable. ${ }^{6}$ In an analysis of the Society of Thoracic Surgeons Database, of 3319 patients with surgically managed cIIIA-N2 disease, $1535(46 \%)$ had induction therapy. ${ }^{25}$ Operative mortality was $2.5 \%$, which is also consistent with a recently published, single-institution ${ }^{10}$ and randomized trial of induction therapy with and without radiation therapy. ${ }^{26}$

These low mortality rates may continue with neoadjuvant immunotherapy. Yang and colleagues ${ }^{27}$ reported a $0 \%$ mortality for 13 patients (10/13 IIIA) given neoadjuvant ipilimumab with a platinum doublet in the TOP1201 trial and no difference in morbidity rates from those previously reported in other preoperative chemotherapy trials. Despite these encouraging results, future studies will need to define morbidity and mortality as it relates to the addition of immunotherapies and toxicities that were well described previously. ${ }^{4}$

Definitive chemoradiotherapy treatment mortalities, however, have remained approximately $2 \%$, and this has not changed since the Albain trial. ${ }^{6}$ Curiously, in the PACIFIC trial, death owing to adverse events occurred in $4.4 \%$ of patients in the durvalumab group and $5.6 \%$ of patients in the placebo group.

\section{CAVEAT 3}

Thoracic surgical and postoperative strategies have been successful since the INT0139 trial in decreasing mortality for patients having induction for IIIA disease. Early experience, specifically with induction immunotherapy in this population of surgical patients, although promising, demands concentrated efforts to minimize toxicity and to document efficacy going forward.

\section{Induction Surgical Stage IIIA Trials Must Have Their OWN Niche}

PACIFIC was an adjuvant immunotherapy trial in stage IIIA disease, and the emphasis of this expert opinion has been on trying to justify induction trials of immunotherapy. Nevertheless, the possibilities for novel N2specific NSCLC protocols involving surgery include studies involving better selection of candidates and sequencing of immunotherapy. Preclinical breast cancer murine models with metastatic potential have reported that neoadjuvant checkpoint inhibition in combination with costimulatory molecules results in significantly longer survival and cures than the use of the combination in an adjuvant setting. ${ }^{28}$ These cures also occur if the surgery is delayed. Early results from a human trial of neoadjuvant combination checkpoint inhibition before resection of high-risk melanoma suggests that neoadjuvant immunotherapy may be superior to adjuvant therapy with greater expansion of $\mathrm{T}$ cell clones in the neoadjuvant arm. ${ }^{29}$ In light of PACIFC, the adjuvant use of immunotherapy should absolutely be exploited in patients with resectable stage IIIA disease; however, thoracic surgeons should be aware of the animal models discussed earlier and should insist that their role in stage IIIA disease in the future will not be reduced to referring patients for adjuvant immunotherapy because they were found to have unsuspected stage IIIA, N2 disease. Thoracic surgeons, at least the $50 \%$ or so of them who have an open mind regarding resection in stage IIIA cancer with only $3 \mathrm{~cm}$ of nodal disease, need to emphasize that future trials must be standalone phase II studies of N2 IIIA disease, which combines modern staging (endobronchial ultrasound, endoesophageal ultrasound, and/or mediastinoscopy) with state-of-the-art imaging (eg, PET-CT) and perhaps novel imaging in the evaluation of these individuals. Pretreatment and ideally preinvasive staging blood-based immunophenotyping should be planned for all patients. These collections are invaluable and should be performed in collaboration with immunology COREs so that hints regarding the degree of surgically harvested posttreatment responses can be possibly intimated from preoperative fluorescence-activated cell sorting or expression analyses. Frankly, we are at the discovery phase with such lofty expectations, and the only way to have the statistical power to lock down these findings will be to design trials that involve only $\mathrm{N} 2$ surgical candidates with novel neoadjuvant or adjuvant immunotherapies. Every surgical stage should be involved with immunotherapy, of course; however, to prevent stage IIIA from becoming a surgical stepchild, we need to increase the portfolio of trials in stage IIIA potentially resectable disease (Table 1), and hopefully a few are under development in the cooperative groups.

\section{CONCLUSIONS}

This so-called expert's opinion is not meant to be confrontational to our nonsurgical partners in the stage IIIA war. It is the plea of an impatient surgeon to help the thoracic oncology community accrue patients for these incredibly important trials. Expert opinions, including those of worldrecognized experts, although well meaning, may be misinterpreted by others, both academic and nonacademic, as "the future is now" in regard to a milestone publication. Patient accrual is tough enough for these types of trials, and it will continue to be so in the future. At least consider giving equipoise a chance for these patients; the contributions of the surgeons could be greater than you think.

\section{Conflict of Intertest Statement}

Author has nothing to disclose with regard to commercial support. 


\section{References}

1. Antonia SJ, Villegas A, Daniel D, Vicente D, Murakami S, Hui R, et al. Durvalumab after chemoradiotherapy in stage III non-small cell lung cancer. $N$ Engl J Med. 2017;377:1919-29.

2. Rizvi NA, Peters S. Immunotherapy for unresectable stage III non-small cell lung cancer. N Engl J Med. 2017;377:1986-8.

3. Guo Y, Krupnick AS. There and back again: an immunotherapy tale. J Thorac Cardiovasc Surg. 2018;155:1771-4.

4. Rusch VW, Chaft J, Hellmann M. KEYNOTE-024: Unlocking a pathway to lung cancer cure? J Thorac Cardiovasc Surg. 2018;155:1777-80.

5. Postmus PE, Kerr KM, Oudkerk M, Senan S, Waller DA, Vansteenkiste J, et al. Early and locally advanced non-small cell lung cancer (NSCLC): ESMO clinical practice guidelines for diagnosis, treatment and follow-up. Ann Oncol. 2017; 28(suppl_4):iv1-21.

6. Albain KS, Swann RS, Rusch VW, Turrisi AT III, Shepherd FA, Smith C, et al. Radiotherapy plus chemotherapy with or without surgical resection for stage III non-small cell lung cancer: a phase III randomised controlled trial. Lancet. 2009; 374:379-86.

7. Andre F, Grunenwald D, Pignon JP, Dujon A, Pujol JL, Brichon PY, et al. Survival of patients with resected N2 non-small cell lung cancer: evidence for a subclassification and implications. J Clin Oncol. 2000;18:2981-9.

8. Ripley RT, Suzuki K, Tan KS, Adusumilli PS, Huang J, Park BJ, et al. Postinduction positron emission tomography assessment of $\mathrm{N} 2$ nodes is not associated with ypN2 disease or overall survival in stage IIIA non-small cell lung cancer. $J$ Thorac Cardiovasc Surg. 2016;151:969-77. 979 e1-3.

9. Rocco G, Nason K, Brunelli A, Varela G, Waddell T, Jones DR. Management of stage IIIA (N2) non-small cell lung cancer: a transatlantic perspective. J Thorac Cardiovasc Surg. 2016;151:1235-8.

10. Kamel MK, Rahouma M, Ghaly G, Nasar A, Port JL, Stiles BM, et al. Clinical predictors of persistent mediastinal nodal disease after induction therapy for stage IIIA N2 non-small cell lung cancer. Ann Thorac Surg. 2017;103: 281-6.

11. Asamura H, Chansky K, Crowley J, Goldstraw P, Rusch VW, Vansteenkiste JF, et al. The International Association for the Study of Lung Cancer lung cancer staging project: proposals for the revision of the $\mathrm{N}$ descriptors in the forthcoming 8th edition of the TNM Classification for Lung Cancer. J Thorac Oncol. 2015;10: 1675-84.

12. Agrawal V, Coroller TP, Hou Y, Lee SW, Romano JL, Baldini EH, et al. Lymph node volume predicts survival but not nodal clearance in stage IIIA-IIIB NSCLC. PLoS One. 2017;12:e174268.

13. Wolchok JD, Hoos A, O'Day S, Weber JS, Hamid O, Lebbe C, et al. Guidelines for the evaluation of immune therapy activity in solid tumors: immune-related response criteria. Clin Cancer Res. 2009;15:7412-20.

14. Seymour L, Bogaerts J, Perrone A, Ford R, Schwartz LH, Mandrekar S, et al. iRECIST: guidelines for response criteria for use in trials testing immunotherapeutics. Lancet Oncol. 2017;18:e143-52.

15. Nishino M, Giobbie-Hurder A, Gargano M, Suda M, Ramaiya NH, Hodi FS. Developing a common language for tumor response to immunotherapy: immune-related response criteria using unidimensional measurements. Clin Cancer Res. 2013;19:3936-43.

16. Solinas C, Porcu M, Hlavata Z, De Silva P, Puzzoni M, Willard-Gallo K, et al. Critical features and challenges associated with imaging in patients undergoing cancer immunotherapy. Crit Rev Oncol Hematol. 2017;120:13-21.

17. Forde PM, Chaft JE, Smith KN, Anagnostou V, Cottrell TR, Hellmann MD, et al. Neoadjuvant PD-1 blockade in resectable lung cancer. $N$ Engl J Med. 2018;378: 1976-86.

18. Van Schil PE. Optimal treatment of stage IIIA-N2 non-small cell lung cancer: a neverending story? J Thorac Oncol. 2017;12:1338-40.

19. Yang CF, Adil SM, Anderson KL, Meyerhoff RR, Turley RS, Hartwig MG, et al. Impact of patient selection and treatment strategies on outcomes after lobectomy for biopsy-proven stage IIIA pN2 non-small cell lung cancer. Eur J Cardiothorac Surg. 2016;49:1607-13.

20. Lim E, McElnay PJ, Rocco G, Brunelli A, Massard G, Toker A, et al. Invasive mediastinal staging is irrelevant for PET/CT positive N2 lung cancer if the primary tumour and ipsilateral lymph nodes are resectable. Lancet Respir Med. 2015;3:e32-3.

21. Darling GE, Li F, Patsios D, Massey C, Wallis AG, Coate L, et al. Neoadjuvant chemoradiation and surgery improves survival outcomes compared with definitive chemoradiation in the treatment of stage IIIA N2 non-small cell lung cancer. Eur J Cardiothorac Surg. 2015;48:684-90; discussion 690.

22. Lee J, Kim HK, Park BJ, Cho JH, Choi YS, Zo JI, et al. Recurrence dynamics after trimodality therapy (neoadjuvant concurrent chemoradiotherapy and surgery) in patients with stage IIIA (N2) lung cancer. Lung Cancer. 2018;115:89-96.

23. Sakib N, Li N, Zhu X, Li D, Li Y, Wang H. Effect of postoperative radiotherapy on outcome in resectable stage IIIA-N2 non-small cell lung cancer: an updated meta-analysis. Nucl Med Commun. 2018;39:51-9.

24. Willers H, Stinchcombe TE, Barriger RB, Chetty IJ, Ginsburg ME, Kestin LL, et al. ACR Appropriateness Criteria(R) induction and adjuvant therapy for N2 non-small cell lung cancer. Am J Clin Oncol. 2015;38:197-205.

25. Boffa D, Fernandez FG, Kim S, Kosinski A, Onaitis MW, Cowper P, et al. Surgically managed clinical stage IIIA-clinical N2 lung cancer in the Society of Thoracic Surgeons database. Ann Thorac Surg. 2017;104:395-403.

26. Pless M, Stupp R, Ris HB, Stahel RA, Weder W, Thierstein S, et al. Induction chemoradiation in stage IIIA/N2 non-small cell lung cancer: a phase 3 randomised trial. Lancet. 2015;386:1049-56.

27. Yang CJ, McSherry F, Mayne NR, Wang X, Berry MF, Tong B, et al. Surgical outcomes after neoadjuvant chemotherapy and ipilimumab for non-small cell lung cancer. Ann Thorac Surg. 2018;105:924-9.

28. Liu J, Blake SJ, Yong MC, Harjunpaa H, Ngiow SF, Takeda K, et al. Improved efficacy of neoadjuvant compared to adjuvant immunotherapy to eradicate metastatic disease. Cancer Discov. 2016;6:1382-99.

29. Rozeman EA, Fanchi L, van Akkooi ACJ, Kvistborg P, Thienen JV, Stegenga B, et al. 1221PD(Neo-)adjuvant ipilimumab + nivolumab (IPI+NIVO) in palpable stage 3 melanoma - updated relapse free survival (RFS) data from the OpACIN trial and first biomarker analyses. Ann Oncol. 2017;28(suppl 5):mdx377.008$\operatorname{mdx} 377.008$. 